\title{
MINIREVIEW
}

\section{Scale-eating cichlids: from hand(ed) to mouth}

\author{
A Richard Palmer* \\ See research article http://www.biomedcentral.com/1741-7007/8/8.
}

\begin{abstract}
Two recent studies in BMC Biology and Evolution raise important questions about a textbook case of frequency-dependent selection in scale-eating cichlid fishes. They also suggest a fascinating new line of research testing the effects of handed behavior on morphological asymmetry.
\end{abstract}

The twisted smiles of left- and right-bending scale-eating cichlids (Figure 1) have achieved near-legendary status among evolutionary biologists since their explosion onto the scene in 1993 [1]. As more data have accumulated, though, the story has become both more interesting and decidedly more puzzling. Two recent papers, by Stewart and Albertson in BMC Biology [2] and Van Dooren et al. in Evolution [3], add valuable new perspectives, including the exciting possibility that handed behavior may amplify mouth asymmetry during growth. But these and other studies also raise important questions about this textbook case of frequency-dependent selection.

The original story [1] is inescapably seductive because it 'makes sense' and is easy to tell. Perissodus microlepis is a specialized scale-eater from Lake Tanganyika that nips scales off the posterior flanks of larger prey fish. Their mouths bend to one side of the head, which allows them to strike from a more posterior orientation that makes them less visible to intended victims. Mouths bend to the right in some individuals and to the left in others, and a single locus, two-allele polymorphism is thought to control the direction of mouth-bending, with right bending being dominant. Finally, frequencies of right- and left-bending individuals appear to vary cyclically around 50:50 over time, as if negative frequency-dependent selection were maintaining this polymorphism. So, the logic goes, when right-bending individuals become more common,

\footnotetext{
${ }^{*}$ Correspondence: rich.palmer@ualberta.ca

Systematics and Evolution Group, Department of Biological Sciences, University of Alberta, Edmonton, AB T6G 2E9, Canada
}

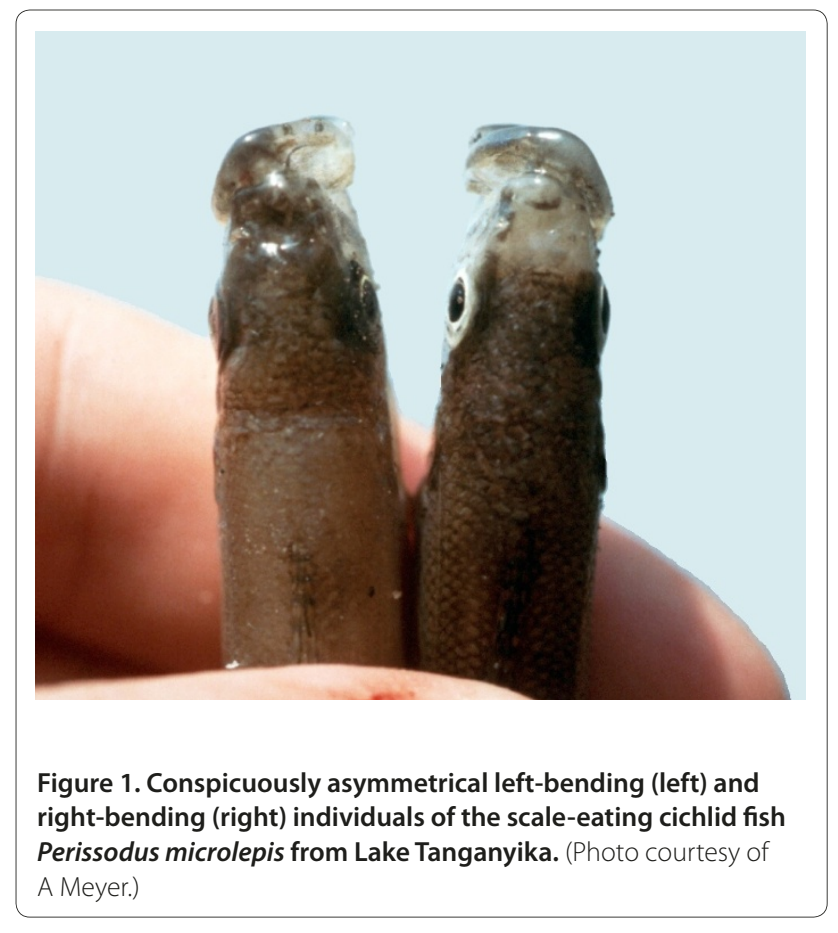

potential victims are thought to become more watchful of their left sides, thereby increasing the feeding success and therefore the fitness of the rarer, left-bending individuals. It's a lovely package.

But puzzling results regarding the extent of mouth bending in adults and juveniles, and its genetic basis, have emerged from several subsequent studies. So the real story may not be so simple.

\section{Extent of mouth-bending in adults and juveniles}

Differences between right- and left-bending morphs of adult $P$. microlepis can sometimes be dramatic (Figure 1). However, careful geometric morphometric analyses of head and jaw asymmetry by Stewart and Albertson [2] and Van Dooren et al. [3] suggest that the differences are more quantitative than qualitative. These new results help to explain why a revision of the genus Perissodus reported conspicuous asymmetry in only one species, P. eccentricus (thought at the time to be a sister species to P. microlepis [4]), and are also consistent with earlier 
quantitative evidence for subtle (2-3\%) and more continuous variation in mouth asymmetry [5].

Among six species of scale-eating cichlids, including P. microlepis, geometric morphometric measures of overall head-shape asymmetry (when viewed laterally) were only significant in adults of one of the two most derived species, P. straeleni [2]. Measurements of lower jaw mechanical advantage differed between right- and left-bending forms in the two most derived scale-eaters, but these differences were only marginally significant for three of the four tests. So, although the four most derived species eat scales almost exclusively, the statistical signal for asymmetry in the heads and jaws of adults appears surprisingly weak.

Similarly, when viewed from above, the mouth-bending angle also appears to be less discrete than generally believed [3]. Careful head-orientation measures of live, adult $P$. microlepis confirmed that some bent towards the right and others to the left. But the frequency distribution of asymmetry values was only weakly bimodal, with modes corresponding to bending angles of 2-3 degrees. Here again, right and left bending did not appear to be discrete states in adults, and more then $10 \%$ of individuals were nearly symmetrical (mouth bend less than 1 degree). As Van Dooren et al. note "mouth bend asymmetry is difficult to determine by visual inspection alone" [3].

Finally, temporal variation in the proportions of leftand right-bending $P$. microlepis is much less pronounced than generally believed. Unlike for total population size, where a confidence limit is difficult to estimate, confidence limits to the proportions of right- and left-bending individuals within a sample are easily obtained from a binomial distribution. Of 13 samples taken over an 11-year period (see Figure 2A of [1]), only two approached statistical significance. Furthermore, in all three populations where two samples were taken concurrently, one yielded an excess of left-bending individuals and the other a deficit. In other words, variation among samples, and therefore among years, was not significantly greater than expected due to simple sampling error.

Mouth-bending is even less pronounced in juvenile P. microlepis. The quantitative analyses of head-bending variation among brooded offspring from a single female [2] yielded two puzzling observations. First, "only 93 (of 141) animals showed unambiguous jaw laterality". Second, although some individuals departed from symmetry by several degrees, the distribution of jaw bending angles was clearly unimodal and centered on zero (see Figure $4 \mathrm{C}$ of [2]). If two alleles at a single locus induce discrete right- and left-bending morphs [1,6], then two questions arise. Why do Stewart and Albertson [2] find that so many juveniles (close to one-third) exhibit no significant mouth bending? And why does asymmetry in juveniles not materialize as a bimodal distribution of right-left differences?

\section{The genetics of mouth bending}

To complicate matters further, the genetic basis of mouth-bending has become more rather than less puzzling as more data accumulate. Two alleles, $R$ and $L$, at a single locus are thought to direct bending to the right or the left, respectively $[1,6]$. But two conflicting models have been advanced. The first is a simple model where $R$ is dominant [1] (Figure 2, Model I), whereas the second is a more complex model, where $R$ is both dominant and homozygous lethal [6] (Figure 2, Model II). Neither model fits all the data. Published offspring frequencies from four crosses are inconsistent with Model I (Figure 2, orange and blue boxes,) and those from six crosses are inconsistent with Model II (Figure 2, yellow and blue boxes). Furthermore, none of the pooled results from the original study [1] (Figure 2a) is consistent with Model II, a model where $R R$ homozygotes are lethal so all rightbending parents must be heterozygotes.

Additional puzzling results emerge from two genetic marker studies. In the first, Stewart and Albertson [2] measured mouth asymmetry quantitatively in juveniles and genotyped them for two microsatellite markers, A and $\mathrm{B}$, putatively linked to right-bending $(R)$ and leftbending $(L)$ alleles, respectively. Their results are not consistent with either of the proposed genetic models. First, nearly half of left-bending juveniles (20 of 45) carried marker A, a result inconsistent with the dominance of allele $R$. Second, nearly 10\% of individuals (9 of 93), regardless of mouth bend, were homozygous for marker A, a result inconsistent with $R R$ lethality (Figure 2, Model II). Third, over $12 \%$ of right-bending juveniles (6 of 48) were homozygous for marker B, a result inconsistent with being homozygous recessive for allele $L$. Finally, although both parents of this brood were inferred to be heterozygotes, and other microsatellite evidence pointed to a single male parent, equal frequencies of rightand left-bending offspring are not consistent with either Model I or II (Figure 2c). In the second genetic marker study, which used both nuclear and mitochondrial DNA markers, Lee et al. [7] report widespread interbreeding between right- and left-bending morphs, consistent with random mating. However, these results are not consistent with the predictions of Model II [6], or with published reports [8], that right-bending males should or do prefer to mate with left-bending females and vice versa (disassortative mating) because $R R$ homozygotes are lethal.

Offspring frequencies are also inconsistent with two other possible models (Figure 2). Cichlid mouth asymmetry might be inherited like visceral asymmetry in mice [9] (that is, like left-right asymmetry of internal organs such as the heart), where a dominant allele causes mouth bending towards one side but a recessive allele yields random phenotypes (equal numbers of right and left) (Figure 2, Model III). Alternatively, the direction of 


\begin{tabular}{|c|c|c|c|c|}
\hline Parent phenotypes & $\mathbf{R} \times \mathbf{R}$ & $R \times L$ & $L \times L$ & Reference \\
\hline \multirow[t]{6}{*}{ F1 proportions } & $R: L$ & $R: L$ & $R: L$ & \\
\hline & $23: 2$ & $74: 0$ & $0: 79$ & {$[1]$} \\
\hline & $35: 11$ & $18: 2$ & $0: 29$ & [1] \\
\hline & $39: 15$ & $55: 53$ & $1: 19$ & {$[1]$} \\
\hline & - & $27: 29$ & $15: 79$ & [1] \\
\hline & - & - & $12: 28$ & {$[1]$} \\
\hline \multirow[t]{7}{*}{ (a) Pooled: } & $97: 28$ & $174: 84$ & 28:234 & {$[1]$} \\
\hline & $71: 40$ & $65: 59$ & $0: 263$ & [6] \\
\hline & $228: 118$ & $44: 36$ & $0: 76$ & {$[6]$} \\
\hline & $95: 37$ & $65: 71$ & $0: 140$ & {$[6]$} \\
\hline & 149:66 & $186: 201$ & - & {$[6]$} \\
\hline & $99: 46$ & $80: 86$ & - & [6] \\
\hline & $67: 38$ & $66: 83$ & - & {$[6]$} \\
\hline (b) Pooled: & 709:385 & $506: 536$ & $0: 479$ & {$[6]$} \\
\hline (c) Total: & $48: 45$ & - & - & [2] \\
\hline Expected proportions & $R: L$ & $R: L$ & $R: L$ & \\
\hline $\begin{array}{l}\text { Model I: two alleles }(R, L), R \\
\text { dominant, no lethality }\end{array}$ & $\begin{array}{l}1: 0 \\
\text { (if } R R \times R L \text { ) } \\
3: 1 \\
\text { (if } R L \times R L \text { ) }\end{array}$ & $\begin{array}{l}1: 0 \\
\text { (if } R R \times L L \text { ) } \\
1: 1 \\
\text { (if } R L \times L L \text { ) }\end{array}$ & $0: 1$ & {$[1]$} \\
\hline $\begin{array}{l}\text { Model II: two alleles }(R, L) \text {, } \\
R \text { dominant, } R R \text { lethal }\end{array}$ & $2: 1$ & $1: 1$ & $0: 1$ & {$[6]$} \\
\hline $\begin{array}{l}\text { Model III: two alleles }(R, N) \\
R \text { dominant, and } N \text { is random }\end{array}$ & $\begin{array}{l}1: 0 \\
\text { (if } R R \times R M \text { ) } \\
7: 1 \\
\text { (if } R N \times R M \text { ) }\end{array}$ & $\begin{array}{l}3: 1 \\
\text { (if } R N \times N N \text { ) } \\
1: 0 \\
\text { (if } R R \times N N \text { ) }\end{array}$ & $\begin{array}{l}1: 1 \\
(\text { if } N N \times N M)\end{array}$ & \\
\hline $\begin{array}{l}\text { Model IV: head-bend } \\
\text { direction is random }\end{array}$ & $1: 1$ & $1: 1$ & $1: 1$ & \\
\hline
\end{tabular}

Figure 2. Parent phenotypes, offspring phenotypes, and expected frequencies of right-bending (R) and left-bending (L) forms of the scale-eating cichlid fish Perissodus microlepis. Each box represents offspring from a single cross (except rows (a) and (b), where results are pooled from all crosses in that study). Offspring were obtained as broods defended by brooding parents in the field. Parent and offspring phenotypes were scored visually, except in row (c) where offspring phenotypes were measured and parent phenotypes inferred from putative microsatellite markers for right- and left-bending. Colors indicate statistically significant departures from expectations for Model I (orange box), Model II (yellow box), or both Models I and II (blue box). Parent phenotypes follow the original convention of Hori [1], who referred to right- and left-bending as dextral and sinistral. This convention differs from [2], who follow the later convention of referring to right-bending fish as 'lefties' because they attack prey from the left side [6]. In regard to Model II, if the RR genotype is lethal as proposed in [6], then all right-bending parents (R) must be RL heterozygotes.

asymmetry might be purely random and not inherited at all, as observed in 27 of 28 cases of random asymmetries in other organisms (Model IV) [10]. Left-bending offspring from two right-bending parents are either too common for model III or too few for Model IV ( 9 of 10 crosses in both cases; Figure 2). Similarly, right-bending offspring from two left-bending parents are too few for either Model III or IV (Figure 2).
Unfortunately, direction of mouth-bending of both parents and offspring was scored by eye in all but one cross (that in Figure 2c), even though mouth-bending angles can be difficult to detect in adults [3] and are so small as to be 'ambiguous' in more than one-third of fry measured [2]. So the validity of these offspring frequencies is hard to judge. Clearly, more studies like that of Stewart and Albertson [2] are needed to resolve these unsettling inconsistencies. 


\section{Handed behavior and morphological asymmetry}

Despite the questions raised above, these recent studies $[2,3]$ suggest an exciting new dimension to the scaleeating cichlid story. Handed or lateralized behavior may amplify morphological asymmetry via developmental plasticity [11]. Handed behavior seems strong and repeatable in these fish $[1,3]$. Hints that it may influence morphological asymmetry in $P$. microlepis come from several sources. First, mouth asymmetry increases with increasing body size [6], as would be expected if repeated lateralized behavior induced greater mouth asymmetry over time. Second, the frequency distribution of mouth asymmetry is not bimodal in brooded juveniles that have not yet begun to feed on scales, suggesting that scaleeating itself may amplify morphological asymmetry later in life [2]. Third, mouth asymmetry of fish forced experimentally to attack prey from their non-preferred side tended to become less pronounced over time, whereas it increased in those allowed to attack from their preferred side [3]. Fourth, the stronger the lateralized behavior of individual fish, the more pronounced the mouth asymmetry [3]. Finally, cichlids, and many other fish, exhibit plastic jaw form that responds to the diet they experience [12]: side of attack may therefore matter just as much as kind of prey eaten.

Several puzzling aspects of the scale-eating cichlid story could therefore be more easily explained if mouth asymmetry is actually induced or amplified by lateralized behavior. However, some intriguing questions remain. Does consistency of lateralized behavior increase with increasing body size, as expected if individuals learn to become more proficient at attacking one side with increasing age? Does lateralized behavior or mouth asymmetry of fish reared in the laboratory on a diet of free-floating scales become less pronounced compared to fish consistently forced to obtain scales by striking the sides of their prey, as expected if lateralized behaviors are learned and morphological asymmetry is amplified by lateralized behavior? Do offspring from left-bending parents exhibit a greater tendency to attack the right sides of their prey even before mouth asymmetry becomes pronounced, as expected if greater genetic variation exists for the degree of lateralized behavior [13] than for the degree of morphological asymmetry? Do those individuals that attack prey consistently from one side obtain more scales than those that sometimes attack from the right or left, as expected if learned behavior improves feeding performance?

As these examples show, studies of right-left asymmetries offer valuable clues about how development evolves because the questions are clear and tests of developmental mechanisms are possible in many different organisms [10].

\section{Acknowledgements}

The freedom to study many aspects of biological asymmetries has been generously supported by NSERC Canada (Discovery Grant A7245) and a sabbatical leave from the University of Alberta.

Published: 24 February 2010

\section{References}

1. Hori M: Frequency-dependent natural selection in the handedness of scale-eating cichlid fish. Science 1993, 260:216-219.

2. Stewart TA, Albertson RC: Evolution of a unique predatory feeding apparatus: Functional anatomy, development and a genetic locus for jaw laterality in Lake Tanganyika scale-eating cichlids. BMC Bio/ 2010, 8:8.

3. Van Dooren TJ, van Goor HA, van Putten M: Handedness and asymmetry in scale-eating cichlids: antisymmetries of different strength. Evolution 2010, doi:10.1111/j.1558-5646.2010.00977.x

4. Liem KF, Stewart DJ: Evolution of the scale eating cichlid fishes of Lake Tanganyika: A generic revision with a description of a new species. Bull Mus Comp Zool, Harvard 1976, 147:319-350.

5. Hori M: Feeding relationships among cichlid fishes in Lake Tanganyika: Effects of intra- and interspecific variations of feeding behavior on their coexistence. Ecol Int Bull 1991, 19:89-101.

6. Hori M, Ochi H, Kohda M: Inheritance pattern of lateral dimorphism in two cichlids (a scale eater, Perissodus microlepis, and an herbivore, Neolamprologus moorii) in Lake Tanganyika. Zool Sci 2007, 24:486-492.

7. Lee HJ, Pittlik S, Jones JC, Salzburger W, Barluenga M, Meyer A: Genetic support for random mating between left and right mouth-morphs in the dimorphic scale-eating cichlid fish, Perissodus microlepis, from Lake Tanganyika. J Fish Biol 2010, in press.

8. Takahashi T, Hori M: Evidence of disassortative mating in a Tanganyikan cichlid fish and its role in the maintenance of intrapopulation dimorphism. Biol Lett 2008, 4:497-499.

9. Hummel KP, Chapman DB: Visceral inversion and associated anomalies in the mouse. J Hered 1959, 50:9-13.

10. Palmer AR: Symmetry breaking and the evolution of development. Science 2004, 306:828-833.

11. Neufeld CJ, Palmer AR: Learning, developmental plasticity, and the rate of morphological evolution. In: Epigenetics: Linking Genotype and Phenotype in Development and Evolution. Edited by Hallgrímsson B, Hall BK. San Francisco: University of California Press; 2010.

12. Meyer A: Phenotypic plasticity and heterochrony in Cichlasoma managuense (Pisces, Cichlidae) and their implications for speciation in cichlid fishes. Evolution 1987, 41:1357-1369.

13. Bisazza A, Dadda M, Facchin L, Vigo F: Artificial selection on laterality in the teleost fish Girardinus falcatus. Behav Brain Res 2007, 178:29-38.

doi:10.1186/jbiol218

Cite this article as: Palmer AR: Scale-eating cichlids: from hand(ed) to mouth. Journal of Biology 2010, 9:11. 